\title{
Homogenisation Applied to Thermal Radiation in Porous Media
}

\author{
C. M. ROONEY, C. P. PLEASE, and S. D. HOWISON \\ Mathematical Institute, 24-29 St Giles, Oxford OX1 3LB, UK
}

(Received 14 May 2020)

\begin{abstract}
Heat transport in granular and porous media occurs through conduction in the solid and radiation through the voids. By exploiting the separation of lengthscales between the small typical particles or voids and the large size of whole region the method of multiple scales can be applied. For a purely diffusive system this yields a problem on the macroscale with an effective conductivity, deduced by solving a "cell problem" on the microscale. Here we apply the method when radiation and conduction are both present, however care must be taken to correctly handle the integral nature of the radiative boundary condition. Again an effective conductivity is found by solving a "cell problem" which, because of the nonlinearity of radiative transfer, to be solved for each temperature value. We also incorporate modifications to the basic theory of multiple scales in order to deal with the non-local nature of the radiative boundary condition. We derive the multiple scales formulation of the problem and report on numerical comparisons between the homogenised problem and direct solution of the problem. We also compare the effective conductivity to that derived using Maxwell models and effective medium theory.
\end{abstract}

Key Words: Asymptotic homogenisation; radiative heat transfer; integral constraints

2020 Mathematics Subject Classification: 35B27; 35B27; 35R09

\section{Introduction}

The heating of granular and composite materials is prevalent in industry, from coal in metallurgical furnaces to heat shields on spacecrafts. The need to understand the behaviour of such materials is evident. However, the endeavour is complicated by the great discrepancy between the size of the bulk material and the size of the grains or pores within it. This renders direct numerical simulations either infeasible or very timeconsuming, particularly in three dimensions. Hence it is valuable to be able to derive effective properties such as thermal conductivity, applicable directly on the bulk scale, which incorporate relevant information from the microscale without the need for full simulation.

The homogenisation method of multiple scales [15, 17 is commonly used to extract homogeneous effective parameters from heterogeneous media. It is founded upon the separation of the macroscopic and microscopic lengthscales and subsequent treatment 
of the variables describing these lengthscales as being independent. Hence at each point on the macroscale, there is a microscale problem to consider, whose solution leads to an effective thermal conductivity on the macroscale encapsulating the relevant effects of microscopic heterogeneities in a parameter. This simplifies the problem while still capturing the vital contribution of the microscale behaviour.

Homogenisation techniques are widely applied to heterogeneous matter. Pasternak and Mühlhaus [16 present two different homogenisation techniques to study a 1D granular material, namely homogenisation by differential expansion and homogenisation by integral transformation, where the former approach was found to produce accurate solutions to the model. Although the second technique produced an exact solution, it was no simpler than the exact, discrete model. Zhou et al. 25] propose a homogenisation technique to study thermal conduction through granular material in a vacuum, neglecting the effect of radiation, and verified their results against DEM simulations. As well as heat flow, the method of multiple scales has been implemented to model fluid transport in tumors 5 as well as used for the improvement of filters using porosity gradients 6 .

An important thermal phenomenon that is neglected by the previous homogenisation analysis is radiation. In many industrial and natural applications radiation within gasfilled voids between conducting solids has a significant impact on the effective thermal conductivity of composite materials. Examples of these include multilayer thermal insulation systems [22], open-celled metal alloy foams 24] and Earth materials within the mantle [21]. The effective conductivities of alumina, graphite and nickel, each perforated with both spherical and cylindrical voids, were experimentally investigated by Francl and Kingery 7. The findings suggest a dependence upon void orientation, especially when the temperatures exceed $500^{\circ} \mathrm{C}$. Experimental exploration of the conductivity of a variety of porous materials was conducted by Luikov et al. 11]. For some materials the conductivities approach a finite limit at high temperatures, whilst for others, the conductivities appear to increase indefinitely. Thus the behaviour of the effective thermal conductivity with temperature is non-trivial and depends not only on the material of interest but also on the material geometry.

It is well known that radiation is most relevant in high-temperature regimes, where experimental analysis is extremely difficult. This supports the need for informative mathematical models that can accurately predict the effective thermal conductivity of granular and composite materials. However, radiation can be complicated to include in microscopic thermal transport models. Typically radiative transport from one solid surface to another through a gas depends on the temperature and orientation of the surfaces and hence models of this involve a non-local, non-linear, integral condition 13, 14, 23. A well-known approximation for radiative transport is the Rosseland approximation 20] which is valid for an optically thick medium where radiation travels only a short distance before being absorbed or scattered 8, 13, 14. In this case it is possible to convert the integral relation for radiative transport into a diffusion condition, similar to that for heat conduction. This approximation provides a significant simplification as energy transport then depends only on the local conditions, and can be accounted for by an effective local conductivity which can be easily studied using standard numerical techniques. However, this approximation is limited to optically thick media and this hinders its application to general composites. 
Tiihonen 23] studied radiation on non-convex surfaces and proved the existence and uniqueness of the conductive-radiative problem. Kiradjiev et al. [10] generalised Maxwell models to account for radiative transfer for a dilute void distribution. They derived a relationship between the effective thermal conductivity and temperature and concluded that the effective thermal conductivity saturates with increasing temperature, or rather the rate of increase of the conductivity slows down as the temperature increases for the dilute distribution.

Homogenisation methods have been applied to conductive-radiative thermal transfer in porous materials. Allaire, El Ganaoui and Habibi [1-3] study thermal transfer within nuclear reactors, more specifically, conductive and radiative transfer through periodic, perforated domains. The authors conduct their homogenisation on the variational formulation of the problem, rather than the strong form. The effective conductivity deduced from this analysis is the same as that derived in the present work; however, we conduct the multiple scales analysis on the strong formulation of the problem. In addition to the value that the two approaches produce consistent results, we also note that there may be cases in which the thermal problem is part of a larger model for which homogenisation of the weak form is not available, whereas our approach is. For example, one may wish to couple the thermal problem with electrical transfer $[19$ and the mechanical properties of granular material. In a forthcoming paper, we describe Ohmic heating due to the presence of electric current, therefore considering the present work using the standard homogenisation framework allows for easy amalgamation with the electrical transfer model.

The non-local nature of the radiative boundary condition poses a complication when applying the method of multiple scales; in particular, one must take care when accounting for integrals on the interfaces between gas and solid. A naïve approach results in an incorrect homogenised model, as discussed by Chapman and McBurnie [4, 12]. Therefore careful adaptation of the multiple scales analysis to allow for slow-scale macroscale variation is necessary properly account for the radiative boundary condition.

In this paper, we apply the method of multiple scales to the strong form of a thermal transport model with radiation in a composite material consisting of a solid perforated with convex voids, with particular focus on the correct representation of the radiative boundary condition in the multiple scales formulation. We begin with a brief outline of how to mathematically model thermal radiation before proposing the full, macroscopic model for thermal transport in a generalised composite material. Following non-dimensionalisation of the model, we discuss in detail how to allow for variation in the slow scale in the integral radiative condition when applying multiple scales analysis, hence extending the standard multiple scales expansion. With all of the necessary components in place, we then proceed by homogenising the thermal transport model and deriving the microscopic cell problem, effective thermal conductivity and homogenised macroscopic model of which this effective conductivity is a parameter. We validate the model numerically by comparing it with a direct simulation of the full problem, focusing on the computational time of the simulations as well as the approximation error between the homogenised and direct solutions. The remainder of the paper is focused on understanding the effect of the gaseous perforations, in particular how their size affects the contribution of radiation to the effective conductivity of the homogenised material. 


\section{Modelling thermal radiation}

Radiative transport between surfaces depends on the orientation of the surfaces relative to each other as well as their temperatures and radiative properties. Here we adopt the standard approach to modelling this effect (see, for example, $[13$ ). By the StefanBoltzmann law, the radiative flux emitted per unit area at a point $\mathbf{x}$ on a void surface $\Gamma$ is

$$
q_{\text {emitted }}(\mathbf{x})=\epsilon_{0} \sigma T^{4}(\mathbf{x}),
$$

where $\epsilon_{0}$ is the surface emissivity, $\sigma$ is the Stefan-Boltzmann constant and $T(\mathbf{x})$ is the absolute temperature at $\mathbf{x} \in \Gamma$. The incident flux on $\mathbf{x}$ from all points $\mathbf{x}^{\prime}$ elsewhere on the surface $\Gamma$ is given by

$$
q_{\text {incident }}(\mathbf{x})=\int_{\Gamma} \epsilon_{0} \sigma T^{4}\left(\mathbf{x}^{\prime}\right) F\left(\mathbf{x}, \mathbf{x}^{\prime}\right) \mathrm{d} S\left(\mathbf{x}^{\prime}\right),
$$

where $F\left(\mathbf{x}, \mathbf{x}^{\prime}\right)$ is known as the view-factor for the surface $\Gamma$; it is a geometrical property which accounts for the effects of surface orientation and separation on radiative transfer. As depicted in Figure 1, $F\left(\mathbf{x}, \mathbf{x}^{\prime}\right)$ quantifies the proportion of the radiation emitted from point $\mathbf{x}^{\prime}$ that strikes point $\mathbf{x}$ and is defined as

$$
F\left(\mathbf{x}, \mathbf{x}^{\prime}\right)=\frac{\cos \theta\left(\mathbf{x}^{\prime}, \mathbf{x}\right) \cos \theta^{\prime}\left(\mathbf{x}^{\prime}, \mathbf{x}\right)}{\pi\left|\mathbf{x}^{\prime}-\mathbf{x}\right|^{2}}
$$

where $\theta\left(\mathbf{x}^{\prime}, \mathbf{x}\right)$ is the angle between the surface normal at $\mathbf{x}^{\prime}$ and the line of sight from $\mathbf{x}^{\prime}$ to $\mathbf{x}$, and $\theta^{\prime}\left(\mathbf{x}^{\prime}, \mathbf{x}\right)$ is the angle between the surface normal at $\mathbf{x}$ and the line of sight. The view factor also accounts for the intensity of radiation at the receiving surface being inversely proportional to the square of the distance from the source. An important property to note is that, for closed convex surfaces, the view factor satisfies

$$
\int_{\Gamma} F\left(\mathbf{x}, \mathbf{x}^{\prime}\right) \mathrm{d} S\left(\mathbf{x}^{\prime}\right)=1 .
$$

Typically in industrial applications involving heat radiation, the material surfaces are not perfectly black, hence some of the radiation hitting the surface is reflected rather than absorbed. For simplicity, we assume that the solid material is a black-body object, thus, the thermal emissivity $\epsilon_{0}=1$. The approach outlined in this paper can be extended to include grey-diffuse surfaces; however, this is not necessary to demonstrate homogenisation of the radiative operator. Furthermore, we also assume that the gas-filled void

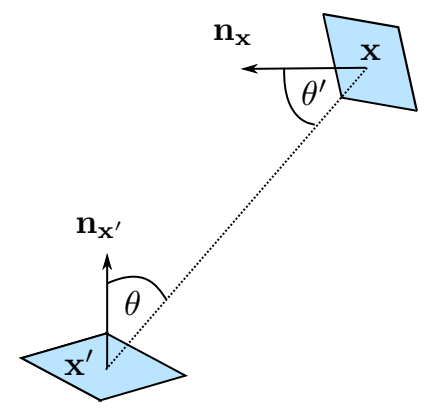

Figure 1. Schematic of two surface elements. 
behaves like a vacuum, namely there is no heat transfer by conduction nor absorption of radiation within the void. An informative review of radiative heat transfer theory can be found in 13 .

\section{Governing equations}

To demonstrate homogenisation by the method of multiple scales of a steady-state conductive-radiative thermal transport model in a composite medium, we consider a simple geometry $\tilde{\Omega}$ of size $L$ perforated by a periodic array of microscopic convex voids. We let the period of the microstructure be $\delta L$, where $\delta \ll 1$. We partition the domain into a solid matrix $\tilde{\Omega}^{S}$ and the $N$ perforations filled with a gas of negligible thermal conductivity. We note that the periodic cell is a cube perforated by one of these voids. We denote the interface between the solid and the gas-filled voids by $\tilde{\Gamma}_{i}$ for $i=1, \ldots, N$. A cross-section of this domain in the case of spherical voids is depicted in Figure 2

We wish to solve the following dimensional system of equations for steady-state temperature $\tilde{T}$ with spatial variable $\tilde{\mathbf{x}}$ within the solid portion of the domain $\tilde{\Omega}^{S}$ with constant thermal conductivity $\tilde{k}$ :

$$
\begin{aligned}
& \tilde{\nabla}^{2} \tilde{T}(\tilde{\mathbf{x}})=0, \quad \tilde{\mathbf{x}} \in \tilde{\Omega}^{S}, \\
& -\tilde{k} \nabla \tilde{T}(\tilde{\mathbf{x}}) \cdot \mathbf{n}=\sigma \tilde{T}^{4}(\tilde{\mathbf{x}})-\int_{\tilde{\Gamma}_{i}} \sigma \tilde{T}^{4}\left(\tilde{\mathbf{x}}^{\prime}\right) F\left(\tilde{\mathbf{x}}, \tilde{\mathbf{x}}^{\prime}\right) \mathrm{d} S\left(\tilde{\mathbf{x}}^{\prime}\right), \quad \tilde{\mathbf{x}} \in \tilde{\Gamma}_{i} \forall i, \\
& \tilde{\nabla} \tilde{T}(\tilde{\mathbf{x}}) \cdot \mathbf{n}=0, \quad \tilde{\mathbf{x}} \in \partial \tilde{\Omega}_{N}, \\
& \tilde{T}(\tilde{\mathbf{x}})=\tilde{T}_{D}\left(\tilde{\mathbf{x}}^{\prime}\right), \quad \tilde{\mathbf{x}} \in \partial \tilde{\Omega}_{D},
\end{aligned}
$$

where $\tilde{T}_{D}(\tilde{\mathbf{x}})$ is a given function on the "Dirichlet" portion of the outer boundary $\partial \tilde{\Omega}_{D}=$

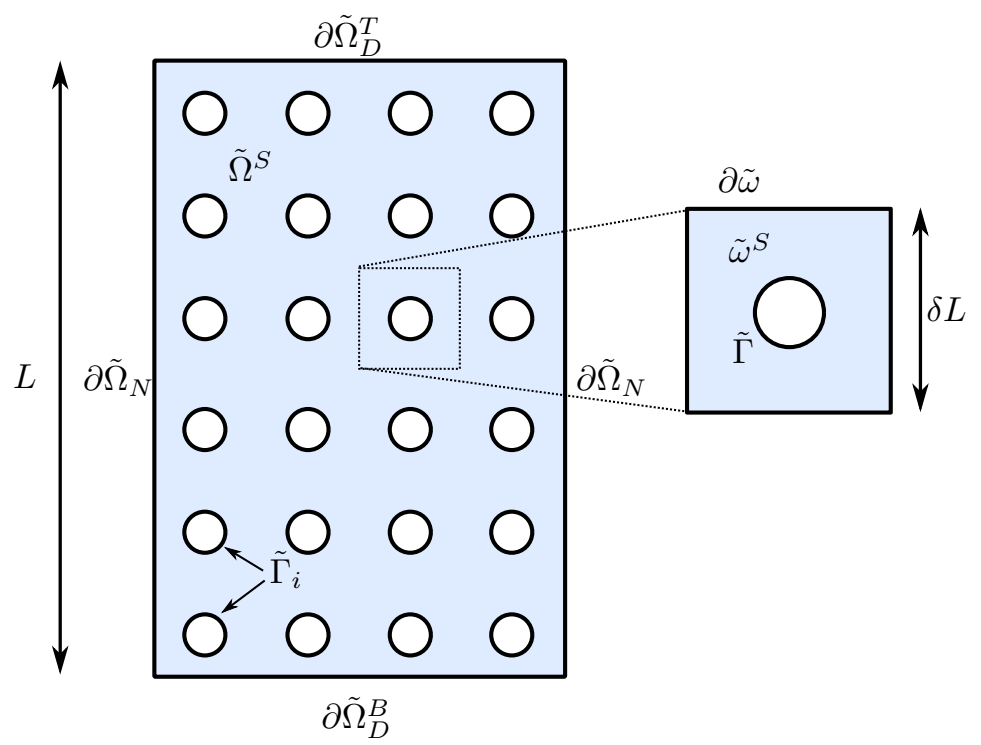

Figure 2. Solid matrix with uniformly distributed spherical perforations in which we solve heat transfer problem. 
$\partial \tilde{\Omega}_{D}^{T} \cup \partial \tilde{\Omega}_{D}^{B}$ and the remainder of the outer boundary is denoted $\partial \tilde{\Omega}_{N}$ and $\mathbf{n}$ is the unit normal pointing into the solid.

For the purposes of motivating our nondimensional variables we consider a simple external boundary condition by defining the function $\tilde{T}_{D}(\tilde{\mathbf{x}})$ as

$$
\tilde{T}_{D}(\tilde{\mathbf{x}})= \begin{cases}T_{M}, & \tilde{\mathbf{x}} \in \partial \tilde{\Omega}_{D}^{T}, \\ T_{m}, & \tilde{\mathbf{x}} \in \partial \tilde{\Omega}_{D}^{B}\end{cases}
$$

where $T_{M}$ and $T_{m}$ are prescribed maximum and minimum temperature values respectively.

The radiative boundary condition (3.1) is derived from the conservation of energy, hence is defined as the balance between the flux emitted (2.1), the incident flux 2.2 and the diffusive flux.

\subsection{Dimensionless equations}

We scale our variables using

$$
\tilde{\mathbf{x}}=L \mathbf{x}, \quad \tilde{T}=T_{M} T,
$$

recalling that $L$ is the lengthscale of $\tilde{\Omega}$ and $T_{M}$ is the maximum temperature values respectively. Therefore, the radiative boundary condition (3.1) becomes

$$
-\nabla T(\mathbf{x}) \cdot \mathbf{n}=\frac{\sigma T_{M}^{3} L}{k}\left(T^{4}(\mathbf{x})-\int_{\Gamma_{i}} T^{4}\left(\mathbf{x}^{\prime}\right) F\left(\mathbf{x}, \mathbf{x}^{\prime}\right) \mathrm{d} S\left(\mathbf{x}^{\prime}\right)\right) .
$$

We want to consider the case where heat transport by conduction and radiation are in balance on the microscale. To deduce the conditions necessary for this balance, we rescale the spatial variable

$$
\mathbf{x}=\delta \mathbf{y}
$$

where $\delta$ is the dimensionless period of the microscale. The dimensionless radiative boundary condition 3.2 becomes

$$
-\frac{1}{\delta} \nabla_{\mathbf{y}} T(\mathbf{y}) \cdot \mathbf{n}=\frac{\sigma T_{M}^{3} L}{k}\left(T^{4}(\mathbf{y})-\int_{\Gamma} T^{4}\left(\mathbf{y}^{\prime}\right) F\left(\mathbf{y}, \mathbf{y}^{\prime}\right) \mathrm{d} S\left(\mathbf{y}^{\prime}\right)\right),
$$

on $\Gamma$, the boundary of the microscopic void and we have introduced the dimensionless parameter

$$
\lambda=\frac{\sigma T_{M}^{3} \delta L}{k} .
$$

We note there are three different regimes that can be captured via $\lambda$.

Regime 1, $\lambda \gg 1$ :

The lowest order balance of 3.3 gives us that

$$
T^{4}(\mathbf{y})=\int_{\Gamma} T^{4}\left(\mathbf{y}^{\prime}\right) F\left(\mathbf{y}, \mathbf{y}^{\prime}\right) \mathrm{d} S\left(\mathbf{y}^{\prime}\right),
$$

for all $\mathbf{y}$ on void boundary $\Gamma$. It can then be shown (a proof is given in Appendix A) that the only solution to this is equation is that $T$ is constant on this boundary. In this 
regime, where radiation dominates on the cell length scale, we can essentially replace the void with a perfect conductor.

Regime 2, $\lambda \ll 1$ :

Here the lowest order balance of 3.3 gives us that

$$
\lambda \ll 1 \Rightarrow \nabla_{\mathbf{y}} T(\mathbf{y}) \cdot \mathbf{n}=0, \quad \mathbf{y} \in \Gamma .
$$

In this regime the void behaves in effect as a perfect insulator and conduction dominates on the cell lengthscale.

Regime 3, $\lambda=O(1)$ :

In this regime we have a balance between conduction and radiation and because this is a distinguished limit, which includes regimes 1 and 2 as special cases, we consider this regime throughout this paper.

For ease of notation, we write 3.2 as

$$
-\nabla T(\mathbf{x}) \cdot \mathbf{n}=\frac{\lambda}{\delta} G_{i}\left(T^{4}\right)(\mathbf{x}), \quad \mathbf{x} \in \Gamma_{i}
$$

where

$$
G_{i}(f)(\mathbf{x})=f(\mathbf{x})-\int_{\Gamma_{i}} f\left(\mathbf{x}^{\prime}\right) F\left(\mathbf{x}, \mathbf{x}^{\prime}\right) \mathrm{d} S\left(\mathbf{x}^{\prime}\right),
$$

and $\Gamma_{i}$ is defined in terms of the macroscopic variable $\mathbf{x}$. We thus write the full, dimensionless problem, with the new radiative boundary condition, as

$$
\begin{aligned}
\nabla^{2} T(\mathbf{x}) & =0, & \mathbf{x} & \in \Omega^{S}, \\
-\nabla T(\mathbf{x}) \cdot \mathbf{n} & =\frac{\lambda}{\delta} G_{i}\left(T^{4}(\mathbf{x})\right)(\mathbf{x}), & & \mathbf{x} \in \Gamma_{i} \forall i, \\
\nabla T(\mathbf{x}) \cdot \mathbf{n} & =0, & & \mathbf{x} \in \partial \Omega_{N}, \\
T(\mathbf{x}) & =T_{D}(\mathbf{x}), & & \mathbf{x} \in \partial \Omega_{D},
\end{aligned}
$$

where the non-dimensional function $T_{D}(\mathbf{x})$ is defined as

$$
T_{D}(\mathbf{x})= \begin{cases}1, & \mathbf{x} \in \partial \Omega_{D}^{T} \\ T_{m} / T_{M}, & \mathbf{x} \in \partial \Omega_{D}^{B}\end{cases}
$$

We note some properties of $G_{i}$ in 3.8 , namely

$$
\begin{array}{ll}
\text { 1. } & G_{i}(f+g)(\mathbf{x})=G_{i}(f)(\mathbf{x})+G_{i}(g)(\mathbf{x}), \\
\text { 2. } & G_{i}(\alpha)(\mathbf{x})=0 \text { for } \alpha \text { independent of } \mathbf{x}, \\
\text { 3. } & \int_{\Gamma_{i}} G_{i}(f)(\mathbf{x}) \mathrm{d} S=0 .
\end{array}
$$

The first of these is linearity which is clear by 3.6 . The second property states that $G_{i}(\cdot)(\mathbf{x})$ is zero when applied to a quantity independent of $\mathbf{x}$ whereas the final property makes use of (2.3) to say that the integral over $G_{i}$ on the appropriate surface $\Gamma_{i}$ is 0 . These identities can be easily proven using $(2.3)$. 
Our challenge in this paper is to consider $(3.7)-(3.9)$ in the limit $\delta \rightarrow 0$ using the multiple scales methodology and to ensure that we carefully account for the radiative boundary condition (3.8). We will see that the standard method is insufficient when dealing with the integral in $G$, thus care must be taken to account for the variation of the macroscale.

\section{Variation of the slow scale}

In standard multiple scales expansions [17, an often ignored issue is that the macroscale variable $\mathbf{x}$ should vary over a single cell. For the vast majority of cases, it is perfectly reasonable to assume a constant value of the macroscale variable when analysing the microscopic cell problem. We recall that the relationship between the macroscale variable $\mathbf{x}$ and microscale variable $\mathbf{y}$ is typically given as $\mathbf{y}=\mathbf{x} / \delta$. Strictly speaking, we actually mean

$$
\mathbf{y}=\mathbf{x} / \delta-\lfloor\mathbf{x} / \delta\rfloor,
$$

where we refer to the component-wise floor function, or alternatively in terms of the component-wise modulo operation

$$
\mathbf{y}=\mathbf{x} / \delta \bmod 1 .
$$

These definitions neglect to account for which periodic cell we are concentrating on, as we are not concerned about the macroscopic position within the domain; only the periodic microscopic behaviour is relevant. For this reason, the slow-scale variable is usually taken to be constant when studying the microscale problem.

However, these definitions are not adequate for the radiative boundary condition 3.8, the principle reason being that the integral condition is non-local. The value of the thermal flux at $\mathbf{x} \in \Gamma_{i}$ depends not only on the temperature at $\mathbf{x}$ but also that on every other point $\mathbf{x}^{\prime} \in \Gamma_{i}$, as indicated by the integral in $G_{i}(\cdot)$. For such non-local behaviour, the slow-scale variable within the periodic cell matters and it cannot be taken to be constant. Further detail can be found in 4,12 .

Before proceeding with the detail of the problem, we highlight the necessity to include variation of the slow scale when dealing with integrals by considering a general condition defined within the microscopic periodic cell.

\subsection{Integral constraints on an internal boundary}

Consider a general integral constraint defined on an internal boundary $\Gamma$ within the periodic cell, such as

$$
\int_{\Gamma} u(\mathbf{x}, \mathbf{y}) \mathrm{d} S(\mathbf{y})=0,
$$

where $\mathbf{x}$ and $\mathbf{y}$ are macroscopic and microscopic points respectively on $\Gamma$ and $u(\cdot, \cdot)$ is a smooth function. We ask whether the condition (4.1) remains unchanged if we use $\mathbf{x}$ or any other point $\hat{\mathbf{x}}$ within the cell; if so, this would allow us to conclude that it is appropriate to use whichever value of the macroscale variable we want within a cell: more 
specifically, we can take $\mathbf{x}$ to be constant. We write

$$
\mathbf{x}=\hat{\mathbf{x}}+\delta(\mathbf{y}-\mathbf{b}),
$$

where $\hat{\mathbf{x}}$ is an arbitrary point within the periodic cell and $\mathbf{b}=\hat{\mathbf{x}} / \delta-\lfloor\hat{\mathbf{x}} / \delta\rfloor$ is the shift between $\hat{\mathbf{x}}$ and the origin of the periodic cell. Asymptotically expanding 4.1) for $\delta \ll 1$, we obtain

$$
\int_{\Gamma}\left\{u_{0}(\hat{\mathbf{x}}, \mathbf{y})+\delta\left[u_{1}(\hat{\mathbf{x}}, \mathbf{y})+(\mathbf{y}-\mathbf{b}) \cdot \nabla_{\mathbf{x}} u_{0}(\hat{\mathbf{x}}, \mathbf{y})\right]+O\left(\delta^{2}\right)\right\} \mathrm{d} S(\mathbf{y})=0,
$$

where we've expanded $u(\mathbf{x}, \mathbf{y})=u_{0}(\mathbf{x}, \mathbf{y})+\delta u_{1}(\mathbf{x}, \mathbf{y})+O\left(\delta^{2}\right)$. At leading order,

$$
\int_{\Gamma} u_{0}(\hat{\mathbf{x}}, \mathbf{y}) \mathrm{d} S(\mathbf{y})=0
$$

which tells us that the leading order expansion of constraint (4.1) is satisfied irrespective of where the macroscale coordinate is evaluated within the periodic cell. Therefore,

$$
\nabla_{\mathbf{x}} \int_{\Gamma} u_{0}(\hat{\mathbf{x}}, \mathbf{y}) \mathrm{d} S(\mathbf{y})=0 .
$$

At $O(\delta)$, we have

$$
\int_{\Gamma} u_{1}(\hat{\mathbf{x}}, \mathbf{y}) \mathrm{d} S(\mathbf{y})=-\int_{\Gamma} \mathbf{y} \cdot \nabla_{\mathbf{x}} u_{0}(\hat{\mathbf{x}}, \mathbf{y}) \mathrm{d} S(\mathbf{y})+\mathbf{b} \cdot \nabla_{\mathbf{x}} \int_{\Gamma} u_{0}(\hat{\mathbf{x}}, \mathbf{y}) \mathrm{d} S(\mathbf{y}) .
$$

By (4.2), the constraint for $u_{1}(\hat{\mathbf{x}}, \mathbf{y})$ is

$$
\int_{\Gamma} u_{1}(\hat{\mathbf{x}}, \mathbf{y}) \mathrm{d} S(\mathbf{y})=-\int_{\Gamma} \mathbf{y} \cdot \nabla_{\mathbf{x}} u_{0}(\hat{\mathbf{x}}, \mathbf{y}) \mathrm{d} S(\mathbf{y}),
$$

which can be evaluated for any value of the macroscale variable $\hat{\mathbf{x}}$ within the periodic cell. Due to this condition, it is clear that

$$
\int_{\Gamma} u(\mathbf{x}, \mathbf{y}) \mathrm{d} S(\mathbf{y})=0 \nRightarrow \int_{\Gamma} u(\hat{\mathbf{x}}, \mathbf{y}) \mathrm{d} S(\mathbf{y})=0,
$$

hence we cannot take the macroscopic variable to be constant when writing an integral in multiple scales form and so we must account for slow-scale variation. We must therefore take extra care when analysing the radiative boundary condition (3.8) as the standard method is not valid.

We continue with the multiple scales analysis of the thermal conductive-radiative problem (3.7)- 3.9).

\section{Extended multiple scales analysis}

We now give a derivation of the homogenised model for the problem where radiation is included. To do this we apply the extended ideas of multiple scales, first to the governing equations and then to the integral condition on the boundary. We perform the expansion of the problem to second order, where a solvability condition gives the leading order homogenised equation. 


\subsection{Multiple scales expansion of heat equation}

We begin by implementing the multiple scales expansion for (3.7), where we set

$$
\mathbf{x}=\hat{\mathbf{x}}+\delta(\mathbf{y}-\mathbf{b}), \quad \nabla \rightarrow \nabla_{\mathbf{x}}+\frac{1}{\delta} \nabla_{\mathbf{y}}
$$

where $\delta$ is the period of the microscale, $\hat{\mathbf{x}}$ is an arbitrary point within the periodic cell and $\mathbf{b}=\hat{\mathbf{x}} / \delta-\lfloor\hat{\mathbf{x}} / \delta\rfloor$ is the shift between $\hat{\mathbf{x}}$ and the origin of the periodic cell. Note that we could consider the standard expansion here, namely $\mathbf{x}=\delta \mathbf{y}$, due to the locality of the equation; however, we use the extended expansion (5.1) to avoid any confusion associated with using different expansions for the heat equation and the radiative boundary condition (3.8). We assume that $T$ has the form

$$
T(\mathbf{x}, \mathbf{y}) \sim T_{0}(\mathbf{x}, \mathbf{y})+\delta T_{1}(\mathbf{x}, \mathbf{y})+\delta^{2} T_{2}(\mathbf{x}, \mathbf{y})+O\left(\delta^{3}\right) .
$$

We then put this into the equation, however for ease of notation, we will use the notation $f=\nabla^{2} T$ so that (3.7) becomes $f=0$. Expanding the terms then gives

$$
f(\mathbf{x}, \mathbf{y})=f(\hat{\mathbf{x}}, \mathbf{y})+\delta(\mathbf{y}-\mathbf{b}) \cdot \nabla_{\mathbf{x}} f(\hat{\mathbf{x}}, \mathbf{y})+\frac{\delta^{2}}{2}(\mathbf{y}-\mathbf{b})^{2} \nabla_{\mathbf{x}}^{2} f(\hat{\mathbf{x}}, \mathbf{y})+\cdots=0,
$$

for $\mathbf{y} \in \omega^{S}$, as depicted in Figure 3 , where we write

$$
f(\hat{\mathbf{x}}, \mathbf{y})=\frac{1}{\delta^{2}} f_{0}(\hat{\mathbf{x}}, \mathbf{y})+\frac{1}{\delta} f_{1}(\hat{\mathbf{x}}, \mathbf{y})+f_{2}(\hat{\mathbf{x}}, \mathbf{y})+O(\delta),
$$

for

$$
\begin{aligned}
& f_{0}(\hat{\mathbf{x}}, \mathbf{y})=\nabla_{\mathbf{y}}^{2} T_{0}, \\
& f_{1}(\hat{\mathbf{x}}, \mathbf{y})=\nabla_{\mathbf{y}}^{2} T_{1}+2 \nabla_{\mathbf{y}} \cdot \nabla_{\mathbf{x}} T_{0}, \\
& f_{2}(\hat{\mathbf{x}}, \mathbf{y})=\nabla_{\mathbf{y}}^{2} T_{2}+2 \nabla_{\mathbf{y}} \cdot \nabla_{\mathbf{x}} T_{1}+\nabla_{\mathbf{x}}^{2} T_{0} .
\end{aligned}
$$

Consider equation 5.2 for each order of $\delta$, namely

$$
\begin{aligned}
O\left(\delta^{-2}\right): & f_{0}=0, \\
O\left(\delta^{-1}\right): & f_{1}+(\mathbf{y}-\mathbf{b}) \cdot \nabla_{\mathbf{x}} f_{0}=0 \quad \Rightarrow \quad f_{1}=0, \\
O(1): & f_{2}+(\mathbf{y}-\mathbf{b}) \cdot \nabla_{\mathbf{x}} f_{1}+\frac{1}{2}(\mathbf{y}-\mathbf{b})^{2} \nabla_{\mathbf{x}}^{2} f_{0}=0 \quad \Rightarrow \quad f_{2}=0 .
\end{aligned}
$$

As expected, this is the same result that we would obtain if we had considered the standard multiple scales expansion, $\mathbf{x}=\delta \mathbf{y}$ in (3.7).

\subsection{Multiple scales expansion of integral constraint}

We expand the integral constraint (3.5) in terms of the two scales using (5.1) by considering the left and right-hand sides separately.

Consider first the left-hand side and introduce the notation $Q=-\nabla T \cdot \mathbf{n}$, so that

$$
Q(\mathbf{x}, \mathbf{y})=Q(\hat{\mathbf{x}}, \mathbf{y})+\delta(\mathbf{y}-\mathbf{b}) \cdot \nabla_{\mathbf{x}} Q(\hat{\mathbf{x}}, \mathbf{y})+\frac{\delta^{2}}{2}(\mathbf{y}-\mathbf{b})^{2} \nabla_{\mathbf{x}}^{2} Q(\hat{\mathbf{x}}, \mathbf{y})+\cdots
$$

for $\mathbf{y} \in \Gamma$, where we write

$$
Q(\hat{\mathbf{x}}, \mathbf{y})=\frac{1}{\delta} Q_{0}(\hat{\mathbf{x}}, \mathbf{y})+Q_{1}(\hat{\mathbf{x}}, \mathbf{y})+\delta Q_{2}(\hat{\mathbf{x}}, \mathbf{y})+\cdots,
$$


where

$$
\begin{aligned}
& Q_{0}=-\nabla_{\mathbf{y}} T_{0} \cdot \mathbf{n}, \\
& Q_{1}=-\left(\nabla_{\mathbf{y}} T_{1}+\nabla_{\mathbf{x}} T_{0}\right) \cdot \mathbf{n}, \\
& Q_{2}=-\left(\nabla_{\mathbf{y}} T_{2}+\nabla_{\mathbf{x}} T_{1}\right) \cdot \mathbf{n} .
\end{aligned}
$$

Thus, the left-hand side of the boundary condition 3.5 for $\mathbf{y} \in \Gamma$ in the unit cell $\omega^{S}$ can be written at each order of $\delta$, namely

$$
\begin{aligned}
O\left(\delta^{-1}\right): \text { LHS } & =-\nabla_{\mathbf{y}} T_{0} \cdot \mathbf{n}, \\
O(1): \quad \text { LHS }=-\left[\left(\nabla_{\mathbf{y}} T_{1}\right.\right. & \left.\left.+\nabla_{\mathbf{x}} T_{0}\right)+(\mathbf{y}-\mathbf{b}) \cdot \nabla_{\mathbf{x}}\left(\nabla_{\mathbf{y}} T_{0}\right)\right] \cdot \mathbf{n}, \\
O(\delta): \quad \text { LHS }=-\left[\left(\nabla_{\mathbf{y}} T_{2}\right.\right. & \left.+\nabla_{\mathbf{x}} T_{1}\right)+(\mathbf{y}-\mathbf{b}) \cdot \nabla_{\mathbf{x}}\left(\nabla_{\mathbf{y}} T_{1}+\nabla_{\mathbf{x}} T_{0}\right) \\
& \left.+\frac{1}{2}(\mathbf{y}-\mathbf{b})^{2} \nabla_{\mathbf{x}}^{2}\left(\nabla_{\mathbf{y}} T_{0}\right)\right] \cdot \mathbf{n} .
\end{aligned}
$$

Now we consider the right-hand side of $(3.5)$, namely

$$
\mathrm{RHS}=\frac{\lambda}{\delta} G_{i}\left(T^{4}\right)(\mathbf{x}) .
$$

and start by considering the integral within $G_{i}(\cdot)(\mathbf{x})$, given by

$$
\mathcal{I}_{i}(\mathbf{x})=\int_{\Gamma_{i}} T^{4}\left(\mathbf{x}^{\prime}\right) F\left(\mathbf{x}, \mathbf{x}^{\prime}\right) \mathrm{d} S\left(\mathbf{x}^{\prime}\right) .
$$

Within the periodic, unit cell $\omega^{S}$ we denote the void boundary by $\Gamma$; therefore we drop the $i$ in $(5.9)$ and write

$$
\mathrm{d} S\left(\mathbf{x}^{\prime}\right)=\delta^{2} \mathrm{~d} S\left(\mathbf{y}^{\prime}\right),
$$

and the right-hand side of 3.5$)$ is then

$$
\begin{aligned}
\mathrm{RHS} & =\frac{\lambda}{\delta} G\left(T^{4}(\mathbf{x}, \mathbf{y})\right)(\mathbf{y}), \\
& =\frac{\lambda}{\delta}\left(T^{4}(\mathbf{x}, \mathbf{y})-\int_{\Gamma} T^{4}\left(\mathbf{x}, \mathbf{y}^{\prime}\right) F\left(\mathbf{y}, \mathbf{y}^{\prime}\right) \mathrm{d} S\left(\mathbf{y}^{\prime}\right)\right) .
\end{aligned}
$$

We note that the integral must be treated as in the general example given in Section 4 .

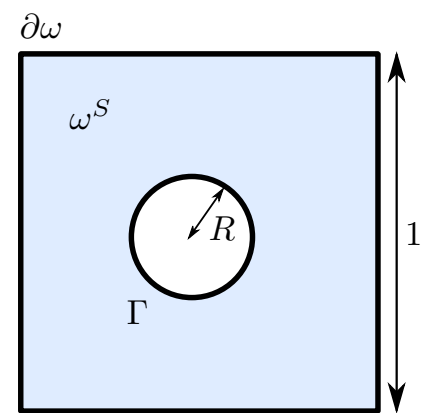

Figure 3. Unit cell in which we solve the cell problem (5.22)-(5.24) 
Using the multiple scales expansion (5.1), we obtain

$$
\begin{aligned}
\operatorname{RHS}=\frac{\lambda}{\delta} G\left(T^{4}(\hat{\mathbf{x}}, \mathbf{y})+\right. & \delta(\mathbf{y}-\mathbf{b}) \cdot \nabla_{\mathbf{x}} T^{4}(\hat{\mathbf{x}}, \mathbf{y}) \\
& \left.+\frac{\delta^{2}}{2}(\mathbf{y}-\mathbf{b})^{2} \nabla_{\mathbf{x}}^{2} T^{4}(\hat{\mathbf{x}}, \mathbf{y})\right)(\mathbf{y})+O\left(\delta^{3}\right) .
\end{aligned}
$$

We thereby write the RHS of 3.5 as

$$
\mathrm{RHS}=\frac{\lambda}{\delta} G\left(\tau_{0}+\delta \tau_{1}+\delta^{2} \tau_{2}+O\left(\delta^{3}\right)\right)(\mathbf{y})
$$

where $\tau_{i}$ are given by

$$
\begin{aligned}
& \tau_{0}=T_{0}^{4}, \\
& \begin{aligned}
\tau_{1}= & 4 T_{0}^{3}\left(T_{1}+(\mathbf{y}-\mathbf{b}) \cdot \nabla_{\mathbf{x}} T_{0}\right), \\
\tau_{2}=2 T_{0}^{2}\left(3 T_{1}^{2}+2 T_{0} T_{2}+\right. & (\mathbf{y}-\mathbf{b}) \cdot\left[6 T_{1} \nabla_{\mathbf{x}} T_{0}+2 T_{0} \nabla_{\mathbf{x}} T_{1}\right] \\
& \left.\quad+(\mathbf{y}-\mathbf{b})^{2}\left[3\left(\nabla_{\mathbf{x}} T_{0}\right)^{2}+T_{0} \nabla_{\mathbf{x}}^{2} T_{0}\right]\right) .
\end{aligned}
\end{aligned}
$$

\subsection{Leading-order problem}

We combine 5.3), 5.6 and 5.10) along with the periodicity of $T_{0}$ to obtain the leadingorder problem, namely

$$
\begin{array}{cl}
\nabla_{\mathbf{y}}^{2} T_{0}=0, & \mathbf{y} \in \omega^{S}, \\
-\nabla_{\mathbf{y}} T_{0} \cdot \mathbf{n}=\lambda G\left(T_{0}^{4}\right)(\mathbf{y}), & \mathbf{y} \in \Gamma, \\
T_{0} \text { periodic. } &
\end{array}
$$

By multiplying 5.12 by $T_{0}$, applying $(5.13)$ and integrating over $\omega^{S}$, we obtain

$$
\int_{\omega^{S}} \nabla_{\mathbf{y}} T_{0} \cdot \nabla_{\mathbf{y}} T_{0} \mathrm{~d} \mathbf{y}+\int_{\Gamma} \lambda G\left(T_{0}^{4}\right)(\mathbf{y}) T_{0} \mathrm{~d} S(\mathbf{y})=0
$$

where the boundary integral over $\partial \omega$ vanishes due to periodicity (5.14). Let us analyse the $\Gamma$-boundary integral in 5.15:

$$
\begin{aligned}
\int_{\Gamma} & \lambda G\left(T_{0}^{4}\right)(\mathbf{y}) T_{0} \mathrm{~d} S(\mathbf{y}) \\
\quad & \lambda \int_{\Gamma}\left(T_{0}^{4}(\cdot, \mathbf{y})-\int_{\Gamma} T_{0}^{4}\left(\cdot, \mathbf{y}^{\prime}\right) F\left(\mathbf{y}, \mathbf{y}^{\prime}\right) \mathrm{d} S\left(\mathbf{y}^{\prime}\right)\right) T_{0}(\cdot, \mathbf{y}) \mathrm{d} S(\mathbf{y}) \\
& =\lambda\left(\int_{\Gamma} T_{0}^{5}(\cdot, \mathbf{y}) \mathrm{d} S(\mathbf{y})-\int_{\Gamma} T_{0}(\cdot, \mathbf{y}) \int_{\Gamma} T_{0}^{4}\left(\cdot, \mathbf{y}^{\prime}\right) F\left(\mathbf{y}, \mathbf{y}^{\prime}\right) \mathrm{d} S\left(\mathbf{y}^{\prime}\right) \mathrm{d} S(\mathbf{y})\right) .
\end{aligned}
$$

We notice the resemblance of (5.16) to the integral Chebyshev inequality, and therefore initially explore bounds of 5.16 .

Lemma 1 For a convex surface $\Gamma$ with view factor $F\left(\mathbf{y}, \mathbf{y}^{\prime}\right) \geqslant 0$ for $\mathbf{y}, \mathbf{y}^{\prime} \in \Gamma$ and $T_{0} \geqslant 0$,

$$
\int_{\Gamma} T_{0}^{5}(\cdot, \mathbf{y}) \mathrm{d} S(\mathbf{y})-\int_{\Gamma} T_{0}(\cdot, \mathbf{y}) \int_{\Gamma} T_{0}^{4}\left(\cdot, \mathbf{y}^{\prime}\right) F\left(\mathbf{y}, \mathbf{y}^{\prime}\right) \mathrm{d} S\left(\mathbf{y}^{\prime}\right) \mathrm{d} S(\mathbf{y}) \geqslant 0 .
$$


Proof of Lemma 1 Consider the inequality

$$
\left(T_{0}^{4}(\cdot, \mathbf{y})-T_{0}^{4}\left(\cdot, \mathbf{y}^{\prime}\right)\right)\left(T_{0}(\cdot, \mathbf{y})-T_{0}\left(\cdot, \mathbf{y}^{\prime}\right)\right) \geqslant 0 .
$$

Multiplying (5.17) by $F\left(\mathbf{y}, \mathbf{y}^{\prime}\right)$ and integrating over $\Gamma$ for $\mathbf{y}$ and $\mathbf{y}^{\prime}$, we deduce

$$
\int_{\Gamma} \int_{\Gamma}\left(T_{0}^{4}(\cdot, \mathbf{y})-T_{0}^{4}\left(\cdot, \mathbf{y}^{\prime}\right)\right)\left(T_{0}(\cdot, \mathbf{y})-T_{0}\left(\cdot, \mathbf{y}^{\prime}\right)\right) F\left(\mathbf{y}, \mathbf{y}^{\prime}\right) \mathrm{d} S\left(\mathbf{y}^{\prime}\right) \mathrm{d} S(\mathbf{y}) \geqslant 0,
$$

from which

$2 \int_{\Gamma} \int_{\Gamma} T_{0}^{5}(\cdot, \mathbf{y}) F\left(\mathbf{y}, \mathbf{y}^{\prime}\right) \mathrm{d} S\left(\mathbf{y}^{\prime}\right) \mathrm{d} S(\mathbf{y})-2 \int_{\Gamma} \int_{\Gamma} T_{0}^{4}\left(\cdot, \mathbf{y}^{\prime}\right) T_{0}(\cdot, \mathbf{y}) F\left(\mathbf{y}, \mathbf{y}^{\prime}\right) \mathrm{d} S\left(\mathbf{y}^{\prime}\right) \mathrm{d} S(\mathbf{y}) \geqslant 0$,

and so

$$
\int_{\Gamma} T_{0}^{5}(\cdot, \mathbf{y}) \mathrm{d} S(\mathbf{y})-\int_{\Gamma} T_{0}(\cdot, \mathbf{y}) \int_{\Gamma} T_{0}^{4}\left(\cdot, \mathbf{y}^{\prime}\right) F\left(\mathbf{y}, \mathbf{y}^{\prime}\right) \mathrm{d} S\left(\mathbf{y}^{\prime}\right) \mathrm{d} S(\mathbf{y}) \geqslant 0,
$$

where we have exploited 2.3 .

Using the Lemma above and returning to the original notation of (5.16), this gives

$$
\int_{\Gamma} \lambda G\left(T_{0}^{4}\right)(\mathbf{y}) T_{0} \mathrm{~d} S(\mathbf{y}) \geqslant 0
$$

and hence the first term on the left-hand side of 5.15 is non-positive, namely

$$
\int_{\omega^{S}} \nabla_{\mathbf{y}} T_{0} \cdot \nabla_{\mathbf{y}} T_{0} \mathrm{~d} \mathbf{y} \leqslant 0
$$

and so

$$
\nabla_{\mathbf{y}} T_{0}=0
$$

We conclude that $T_{0}=T_{0}(\mathbf{x})$ is independent of the microscale $\mathbf{y}$.

\subsection{First-order problem}

We combine 5.4, (5.7) and 5.10), noting the cancellation of the $\nabla_{\mathbf{y}} T_{0}$ terms, to write the first-order problem as

$$
\begin{array}{cl}
\nabla_{\mathbf{y}}^{2} T_{1}=0, & \mathbf{x} \in \omega^{S}, \\
-\left(\nabla_{\mathbf{y}} T_{1}+\nabla_{\mathbf{x}} T_{0}\right) \cdot \mathbf{n}=\lambda G\left(4 T_{0}^{3}\left[T_{1}+(\mathbf{y}-\mathbf{b}) \cdot \nabla_{\mathbf{x}} T_{0}\right]\right)(\mathbf{y}), & \mathbf{x} \in \Gamma, \\
T_{1} \text { periodic. } &
\end{array}
$$

Due to the linearity of 5.18 and 5.19 , we can write $T_{1}$ in the form

$$
T_{1}=\mathbf{\Psi}(\mathbf{y}) \cdot \nabla_{\mathbf{x}} T_{0}(\mathbf{x})+\hat{T}_{1}(\mathbf{x})
$$

where $\mathbf{\Psi}(\mathbf{y})$ is some vector function and $\hat{T}_{1}(\mathbf{x})$ is some scalar function, both to be found. We show below that the lowest order homogenised equation depends only on $\nabla_{\mathbf{y}} T_{1}$ and hence we only need to find $\mathbf{\Psi}(\mathbf{y})$ to obtain the lowest order macroscopic equation. Sub- 
stituting 5.21 into $5.18-5.20$ we obtain the cell problem

$$
\begin{array}{cl}
\nabla_{\mathbf{y}}^{2} \mathbf{\Psi}=0, & \mathbf{x} \in \omega^{S}, \\
-\left(\nabla_{\mathbf{y}} \mathbf{\Psi}+\mathbf{I}\right) \cdot \mathbf{n}=4 \lambda T_{0}^{3} G(\mathbf{\Psi}+\mathbf{y})(\mathbf{y}), & \mathbf{x} \in \Gamma, \\
\mathbf{\Psi} \text { periodic. } &
\end{array}
$$

Note that in writing $(5.23)$ we use a simplification of the right-hand side of $(5.19)$ which exploits 3.10 namely that

$$
\begin{aligned}
G\left(4 T_{0}^{3}\left[T_{1}+(\mathbf{y}-\mathbf{b}) \cdot \nabla_{\mathbf{x}} T_{0}\right]\right)(\mathbf{y}) & =4 T_{0}^{3} G\left(\Psi \cdot \nabla_{\mathbf{x}} T_{0}+(\mathbf{y}-\mathbf{b}) \cdot \nabla_{\mathbf{x}} T_{0}\right)(\mathbf{y}), \\
& =4 T_{0}^{3} G(\mathbf{\Psi}+(\mathbf{y}-\mathbf{b}))(\mathbf{y}) \cdot \nabla_{\mathbf{x}} T_{0} \\
& =4 T_{0}^{3}[G(\mathbf{\Psi}+\mathbf{y})(\mathbf{y})-G(\mathbf{b})(\mathbf{y})] \cdot \nabla_{\mathbf{x}} T_{0} \\
& =4 T_{0}^{3} G(\mathbf{\Psi}+\mathbf{y})(\mathbf{y}) \cdot \nabla_{\mathbf{x}} T_{0} .
\end{aligned}
$$

\subsection{Second-order problem}

Finally, we combine (5.5), 5.8) and (5.10) to obtain the second-order problem, given by

$$
\begin{array}{cc}
\nabla_{\mathbf{y}}^{2} T_{2}+2 \nabla_{\mathbf{y}} \cdot \nabla_{\mathbf{x}} T_{1}+\nabla_{\mathbf{x}}^{2} T_{0}=0, & \mathbf{x} \in \omega^{S}, \\
-\left[\nabla_{\mathbf{y}} T_{2}+\nabla_{\mathbf{x}} T_{1}-(\mathbf{y}-\mathbf{b}) \cdot \nabla_{\mathbf{x}}\left(\nabla_{\mathbf{y}} T_{1}+\nabla_{\mathbf{x}} T_{0}\right)\right] \cdot \mathbf{n}=\lambda G\left(\tau_{2}\right)(\mathbf{y}), & \mathbf{x} \in \Gamma, \\
T_{2} \text { periodic, } &
\end{array}
$$

where $\tau_{2}$ is given by (5.11). The solvability condition for the existence of $T_{2}$ is found by integrating 5.25 over $\omega$ and applying the divergence theorem to obtain

$$
\nabla_{\mathbf{x}} \cdot\left(\frac{1}{|\omega|} \int_{\omega^{S}}\left(\nabla_{\mathbf{y}} T_{1}+\nabla_{\mathbf{x}} T_{0}\right) \mathrm{d} \mathbf{y}\right)+\frac{1}{|\omega|} \int_{\partial \omega^{S}}\left(\nabla_{\mathbf{y}} T_{2}+\nabla_{\mathbf{x}} T_{1}\right) \cdot \mathbf{n} \mathrm{d} S(\mathbf{y})=0
$$

The boundary integral on the left-hand side of (5.27) can be written as the sum of the integral over $\Gamma$ and the integral over $\partial \omega$; however, the latter vanishes by periodicity of $T_{1}$ and $T_{2}$. Therefore we focus on the integral over $\Gamma$. Using (5.26), we can write the boundary integral in (5.27) as

$$
\begin{aligned}
\int_{\Gamma}\left(\nabla_{\mathbf{y}} T_{2}\right. & \left.+\nabla_{\mathbf{x}} T_{1}\right) \cdot \mathbf{n} \mathrm{d} S(\mathbf{y}) \\
& =-\int_{\Gamma}\left[(\mathbf{y}-\mathbf{b}) \cdot \nabla_{\mathbf{x}}\left(\nabla_{\mathbf{y}} T_{1}+\nabla_{\mathbf{x}} T_{0}\right) \cdot \mathbf{n}+\lambda G\left(\tau_{2}\right)(\mathbf{y})\right] \mathrm{d} S(\mathbf{y}) .
\end{aligned}
$$

Note that, from 3.10 , we have

$$
\int_{\Gamma} G\left(\tau_{2}\right)(\mathbf{y}) \mathrm{d} S(\mathbf{y})=0
$$

and from the first-order boundary condition $[5.19]$, that

$$
\begin{aligned}
-\int_{\Gamma}(\mathbf{y}-\mathbf{b}) \cdot \nabla_{\mathbf{x}}\left(\nabla_{\mathbf{y}} T_{1}+\nabla_{\mathbf{x}} T_{0}\right) \cdot \mathbf{n} \mathrm{d} S(\mathbf{y}) \\
=\nabla_{\mathbf{x}} \cdot\left(4 \lambda T_{0}^{3} \int_{\Gamma} \mathbf{y} G\left(T_{1}+\mathbf{y} \cdot \nabla_{\mathbf{x}} T_{0}\right)(\mathbf{y})^{\top} \mathrm{d} S(\mathbf{y})\right) .
\end{aligned}
$$


Therefore, recalling 5.21, 5.27 becomes

$$
\begin{aligned}
\nabla_{\mathbf{x}} \cdot\left(\frac{1}{|\omega|} \int_{\omega^{S}}\left(\nabla_{\mathbf{y}} T_{1}+\nabla_{\mathbf{x}} T_{0}\right) \mathrm{d} \mathbf{y}\right) \\
\quad+\nabla_{\mathbf{x}} \cdot\left(\frac{4 \lambda T_{0}^{3}}{|\omega|} \int_{\Gamma} \mathbf{y} G\left(T_{1}+\mathbf{y} \cdot \nabla_{\mathbf{x}} T_{0}\right)(\mathbf{y})^{\top} \mathrm{d} S(\mathbf{y})\right)=0
\end{aligned}
$$

that is,

$$
\nabla_{\mathbf{x}} \cdot\left(\frac{1}{|\omega|}\left[\int_{\omega^{S}}\left(\mathbf{I}+\nabla_{\mathbf{y}} \mathbf{\Psi}\right) \mathrm{d} \mathbf{y}+4 \lambda T_{0}^{3} \int_{\Gamma} \mathbf{y} G(\mathbf{\Psi}+\mathbf{y})(\mathbf{y})^{\top} \mathrm{d} S(\mathbf{y})\right] \nabla_{\mathbf{x}} T_{0}\right)=0
$$

where the matrix $\mathbf{y} G(\mathbf{\Psi}+\mathbf{y})(\mathbf{y})^{\top}$ has entries

$$
\begin{aligned}
\left(\mathbf{y} G(\Psi+\mathbf{y})(\mathbf{y})^{\top}\right)_{i j} & =y_{i} G\left(\Psi_{j}+y_{j}\right)(\mathbf{y}), \\
& =y_{i}\left(\Psi_{j}+y_{j}-\int_{\Gamma}\left(\Psi_{j}+y_{j}^{\prime}\right) F\left(\mathbf{y}, \mathbf{y}^{\prime}\right) \mathrm{d} S\left(\mathbf{y}^{\prime}\right)\right) .
\end{aligned}
$$

Hence we arrive at the dimensionless homogenised problem

$$
\begin{aligned}
\nabla_{\mathbf{x}} \cdot\left(\boldsymbol{k}_{\text {eff }}\left(T_{0}\right) \nabla_{\mathbf{x}} T_{0}(\mathbf{x})\right) & =0, & & \mathbf{x} \in \Omega, \\
\nabla_{\mathbf{x}} T_{0}(\mathbf{x}) \cdot \mathbf{n} & =0, & & \mathbf{x} \in \partial \Omega_{N}, \\
T_{0}(\mathbf{x}) & =T_{D}(\mathbf{x}), & & \mathbf{x} \in \partial \Omega_{D},
\end{aligned}
$$

where the effective conductivity is given from the microscale by

$$
\boldsymbol{k}_{\mathrm{eff}}\left(T_{0}\right)=\frac{1}{|\omega|}\left(\int_{\omega^{S}}\left(\mathbf{I}+\nabla_{\mathbf{y}} \mathbf{\Psi}\right) \mathrm{d} \mathbf{y}+4 \lambda T_{0}^{3} \int_{\Gamma} \mathbf{y} G(\mathbf{\Psi}+\mathbf{y})(\mathbf{y})^{\top} \mathrm{d} S(\mathbf{y})\right),
$$

and $\boldsymbol{\Psi}$ satisfies the cell problem (5.22)-(5.24). We note that the final integral in $\boldsymbol{k}_{\text {eff }}$ is, perhaps not so obviously, independent of the origin of the cell problem. This can be proven by considering properties of $G$ given by 3.10 .

\subsection{Large $\lambda$ limit}

We stated in Section 3.1 that when $\lambda=O(1)$ we have balance between conduction and radiation on the cell length scale. We now consider the limit $\lambda \rightarrow \infty$ to study the effect of large temperatures on the effective conductivity. Recall the microscopic cell problem given by $5.22-5.24$ and write

$$
4 \lambda T_{0}^{3}=\frac{1}{\epsilon},
$$

so that $\epsilon \rightarrow 0$ as $\lambda \rightarrow \infty$. Expanding $\boldsymbol{\Psi}$ for small $\epsilon$,

$$
\boldsymbol{\Psi}=\boldsymbol{\Psi}_{0}+\epsilon \mathbf{\Psi}_{1}+\epsilon^{2} \boldsymbol{\Psi}_{2}+O\left(\epsilon^{3}\right),
$$

the $O(1)$ cell problem for $\boldsymbol{\Psi}_{0}$ is given by

$$
\begin{aligned}
\nabla_{\mathbf{y}}^{2} \mathbf{\Psi}_{0}=0, & \mathbf{x} \in \omega^{S}, \\
G\left(\boldsymbol{\Psi}_{0}+\mathbf{y}\right)(\mathbf{y})=0, & \mathbf{x} \in \Gamma, \\
\boldsymbol{\Psi}_{0} \text { periodic. } &
\end{aligned}
$$


and the $O(\epsilon)$ problem for $\boldsymbol{\Psi}_{1}$ is

$$
\begin{array}{cc}
\nabla_{\mathbf{y}}^{2} \boldsymbol{\Psi}_{1}=0, & \mathbf{x} \in \omega^{S}, \\
-\left(\nabla_{\mathbf{y}} \mathbf{\Psi}_{0}+\mathbf{I}\right) \cdot \mathbf{n}=G\left(\boldsymbol{\Psi}_{1}\right)(\mathbf{y}), & \mathbf{x} \in \Gamma, \\
\mathbf{\Psi}_{1} \text { periodic. } &
\end{array}
$$

Considering (5.31) and 5.32) in the expression for the effective conductivity (5.30) and using the $O(1)$ boundary condition 5.33 , we write

$$
\boldsymbol{k}_{\mathrm{eff}}=\frac{1}{|\omega|}\left(\int_{\omega^{S}}\left(\mathbf{I}+\nabla_{\mathbf{y}} \mathbf{\Psi}_{0}\right) \mathrm{d} \mathbf{y}+\int_{\Gamma} \mathbf{y} G\left(\mathbf{\Psi}_{1}\right)(\mathbf{y})^{\top} \mathrm{d} S(\mathbf{y})\right)+O(\epsilon) .
$$

We note that the $O(\epsilon)$ boundary condition (5.34) allows us to write this in terms of $\boldsymbol{\Psi}_{0}$, namely

$$
\boldsymbol{k}_{\mathrm{eff}}=\frac{1}{|\omega|}\left(\int_{\omega^{S}}\left(\mathbf{I}+\nabla_{\mathbf{y}} \mathbf{\Psi}_{0}\right) \mathrm{d} \mathbf{y}-\int_{\Gamma} \mathbf{y}\left[\left(\nabla_{\mathbf{y}} \mathbf{\Psi}_{0}+\mathbf{I}\right) \cdot \mathbf{n}\right]^{\top} \mathrm{d} S(\mathbf{y})\right)+O(\epsilon) .
$$

This analysis indicates that the conductivity is bounded as $\lambda \rightarrow \infty$ and furthermore gives an expression 5.35 for the limit. As mentioned previously, in this limit, the void behaves as if it is a perfect conductor, so that the entirety of its boundary is at the same temperature (namely $T_{0}+\delta T_{1}$ ).

\section{Numerical results}

We investigate the efficiency and accuracy of the homogenisation method and explore the influence of radiative transfer on the effective thermal conductivity. We validate the homogenised solution by comparing it with the direct numerical solution of the exact model (3.7)- 3.9$)$ for a range of periods $\delta$ to illustrate convergence, while also comparing the computational cost of the two methods.

For computational ease in the direct simulation, we consider a two-dimensional domain consisting of a square-shaped solid perforated with circular voids, as in Figure 2 For the direct solution to (3.7)- (3.9), we consider a domain $\Omega=[0,1] \times[0,1]$ consisting of solid matrix $\Omega^{S}$ perforated with $N=\delta^{-2}$ circular voids with boundaries $\Gamma_{i}$ and macroscopic radius $\hat{R}=R \delta$ for $R=0.1$. We consider the Dirichlet portion of the external boundary $\partial \Omega_{D}=\partial \Omega_{D}^{T} \cup \partial \Omega_{D}^{B}$ to be

$$
\partial \Omega_{D}^{T}=\{(x, y) \in \partial \Omega: y=1\}, \quad \partial \Omega_{D}^{B}=\{(x, y) \in \partial \Omega: y=0\},
$$

on which we define

$$
T_{D}(\mathbf{x})= \begin{cases}1, & \mathbf{x} \in \partial \Omega_{D}^{T}, \\ T_{m} / T_{M}, & \mathbf{x} \in \partial \Omega_{D}^{B} .\end{cases}
$$

The remainder of the boundary is denoted $\partial \Omega_{N}$, on which zero-flux conditions are enforced. We solve the homogenised problem (5.28)-5.29) in the homogeneous domain $\Omega_{H}=[0,1] \times[0,1]$ with the same external boundary conditions as above.

We recall that our analysis was conducted as $\delta \rightarrow 0$ with $\lambda=O(1)$; therefore, we solve the models for $\lambda=1$. Note that for a two-dimensional disk of radius $R$ with boundary 
$\Gamma$, the view factor between $\mathbf{x}$ and $\mathbf{x}^{\prime}$ on $\Gamma$, captured within $G(\cdot) 3.5$, can be deduced using trigonometry $[8]$ to be

$$
F\left(\mathbf{x}, \mathbf{x}^{\prime}\right)=\frac{1}{2 \pi R},
$$

whereas for a sphere of radius $R$, the view factor is

$$
F\left(\mathbf{x}, \mathbf{x}^{\prime}\right)=\frac{1}{4 \pi R^{2}} .
$$

\subsection{Homogenisation algorithm}

We note the dependence of the cell solution $\boldsymbol{\Psi}$ on the homogenised temperature $T(\mathbf{x})$, indicated by the presence of $T_{0}$ in the cell problem boundary condition 5.23 . Therefore, we must solve the cell problem for all temperature values in order to calculate the effective conductivity (5.30). We can consider a selection of temperature values and interpolate to obtain an approximation of $\boldsymbol{k}_{\text {eff }}$ over the full range of temperatures. We propose the following algorithm for solving the homogenised problem:

(1) solve the cell problem (5.22)-(5.24) for a variety of $T_{0}$;

(2) calculate the effective conductivity tensor $\boldsymbol{k}_{\text {eff }}$ (5.30) for each value of $T_{0}$;

(3) interpolate the effective conductivity values by a cubic spline to obtain an approximation to the function $\boldsymbol{k}_{\text {eff }}\left(T_{0}\right)$ valid for every $T_{0}$ within a given range;

(4) solve the (nonlinear) homogenised problem (5.28)-(5.29) with effective conductivity function $\boldsymbol{k}_{\text {eff }}\left(T_{0}\right)$ for homogenised temperature $T_{0}$.

We solve the cell, homogenised and direct problems all using piecewise quadratic finite elements in Firedrake [18 on a mesh of triangles. We use the Scipy 9 package interpolate to fit the spline to the calculated values of $\boldsymbol{k}_{\text {eff }}\left(T_{0}\right)$.

\subsection{Results}

There is significant numerical complexity associated with implementing the nonlinear condition (3.8) on the void boundary, thus considering large numbers of such voids renders the direct solution infeasible if $\delta$ is very small. Therefore we include results for only $\delta=0.5,0.25,0.125$ to demonstrate convergence of the homogenised solution to the direct

solution and the computational superiority of the former. We note that a greater range of $\delta$ could be considered with increased computational power or numerical sophistication; however, this is beyond the scope of this work. We include only a sample of direct simulations to validate the homogenised solution.

By interpolating the homogenised solution onto the heterogeneous domain $\Omega^{S}$, we calculate the $L^{2}$-error in $\Omega^{S}$ between the homogenised and direct solutions, which we plot against $\delta$ in Figure $4 \mathrm{a}$. We see that the error decreases approximately as $O(\delta)$ as expected, indicating that as $\delta$ decreases, the homogenised solution accurately approximates the "true" solution and can thereby be used to estimate the behaviour of thermal conduction in a solid domain perforated with periodically distributed gas-filled voids. In Figure $4 \mathrm{~b}$, we plot the computational time of solving the problem directly for $\delta$ and see that, in the best case, the computational time scales like $O\left(\delta^{-2}\right)$. 


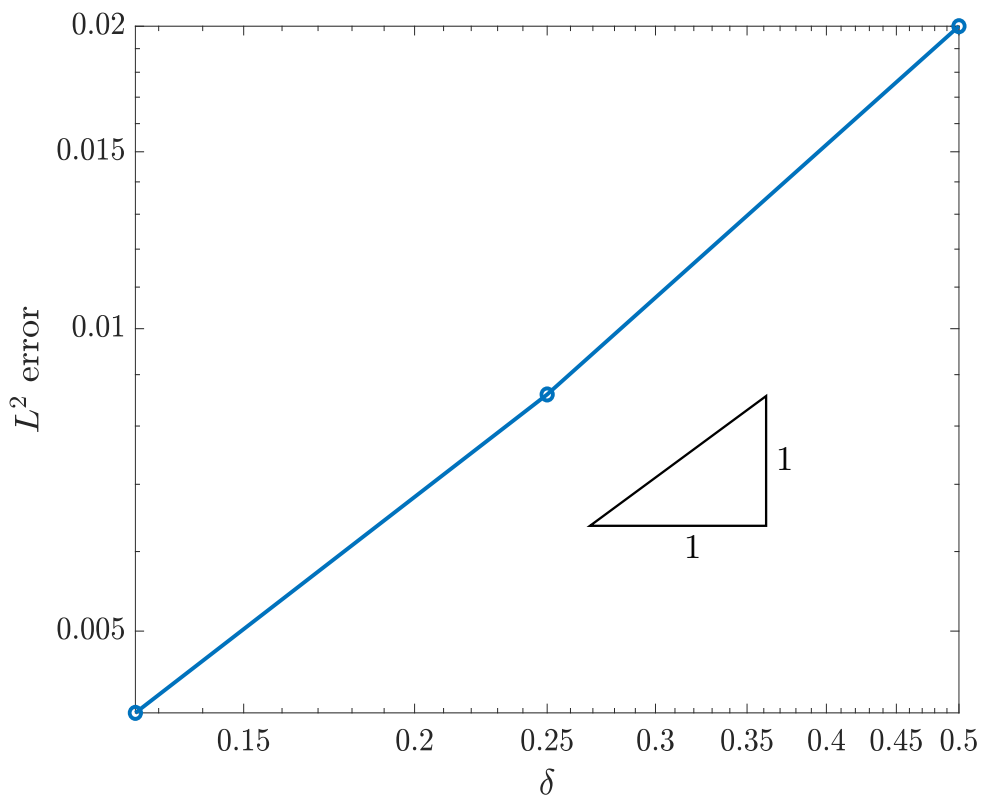

(a) $L^{2}$-error between homogenised solutions and direct solution for different values of $\delta$.

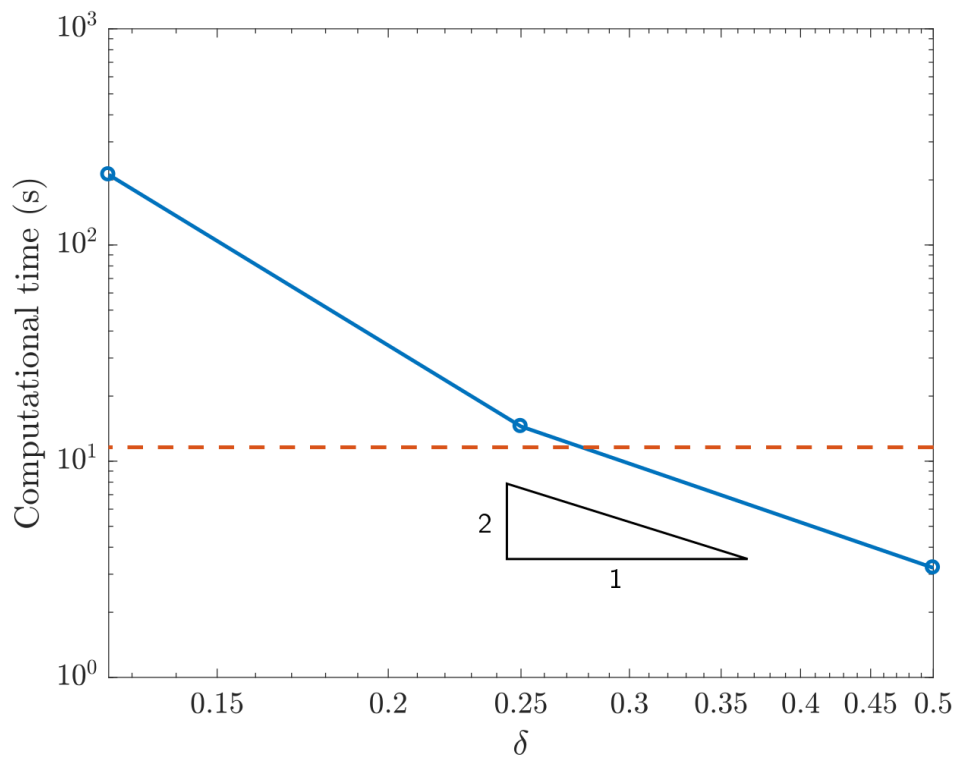

(b) Computational time of solving the problem directly (blue solid line) with $\delta$, along with the time to solve homogenisation problem, namely $11.8 \mathrm{~s}$ (dashed orange line).

Figure 4. $L^{2}$-error and computational time associated with the direct and homogenised solutions. 


\section{Variation of effective conductivity with temperature}

We are interested in how the void radius affects the effective conductivity tensor. Kiradjiev et al. 10 investigated this relationship for effective conductivities derived using Maxwell models, effective medium theory (EMT) and differential effective medium theory (DEMT). The theory and formulation of these models are well explained in [10] . In this section we compare our effective conductivity obtained via homogenisation with those obtained by Kiradjiev. Note that this analysis is conducted in three-dimensions; therefore, we consider a periodic array of spheres within a solid matrix so that our cell domain is a unit cube perforated with a sphere of radius $R$. Furthermore, we must consider the three-dimensional view factor (6.1) within $G(\cdot)$.

For this analysis, we must consider the dimensional effective conductivity $\tilde{\boldsymbol{k}}_{\text {eff }}(\tilde{T})$, given by

$$
\tilde{\boldsymbol{k}}_{\mathrm{eff}}(\tilde{T})=\frac{k}{|\omega|}\left(\int_{\omega^{S}}\left(\mathbf{I}+\nabla_{\mathbf{y}} \mathbf{\Psi}\right) \mathrm{d} \mathbf{y}+\frac{4 \sigma \delta L \tilde{T}^{3}}{k} \int_{\Gamma} \mathbf{y} G(\mathbf{\Psi}+\mathbf{y})(\mathbf{y})^{\top} \mathrm{d} S(\mathbf{y})\right) .
$$

We must define parameters $L$ and $R$ such that our environment is identical to that studied by Kiradjiev et al. who consider a solid domain perforated with spherical gas-voids of average radii $R_{K}=0.01 \mathrm{~m}$ or $0.05 \mathrm{~m}$ and porosity $\phi=0.1$. Note that $\phi$ determines the void spacing. Due to the periodic nature of our domain, the porosity is equal to the porosity of the unit cell, so that we derive $R$ to be

$$
R=\left(\frac{3 \phi}{4 \pi}\right)^{\frac{1}{3}} \approx 0.2879 .
$$

To ensure that our dimensional radius $\delta L R$ is equal to $R_{K}$, we need

$$
\delta L=\frac{R_{K}}{R} .
$$

Finally we take $k=30$ as in [10], noting that we had already considered $\epsilon=1$ throughout our analysis.

The symmetry of the unit cell $\omega$ means that $\tilde{\boldsymbol{k}}_{\text {eff }}$ is a scalar multiple of the identity matrix, namely

$$
\tilde{\boldsymbol{k}}_{\mathrm{eff}}=\tilde{k}_{\mathrm{eff}} \mathbf{I} .
$$

We repeat steps $1-3$ of the homogenisation algorithm for $R_{K}=0.01 \mathrm{~m}$ and $0.05 \mathrm{~m}$ and plot the corresponding effective conductivity scalar multiple $\tilde{k}_{\text {eff }}$ against $\tilde{T}$ in Figure 5 , where $\tilde{T}$ is the dimensional temperature. We also plot the results from Maxwell models, EMT and DEMT presented by Kiradjiev.

Although all four models produce similar trends, we see that the effective conductivity obtained via homogenisation most closely agrees with that of Maxwell. For both void radii, we observe saturation in the effective conductivity at high temperatures, behaviour that is attributed partly to the solid material and is captured by studying the $\lambda \rightarrow \infty$ limit in Section 5.6. As the temperature gets very large, the radiative condition means that the voids act as perfect conductors, thus the solid is the rate-limiting factor. Therefore, due to the geometry, radiation does not dominate over conduction for high temperatures. 


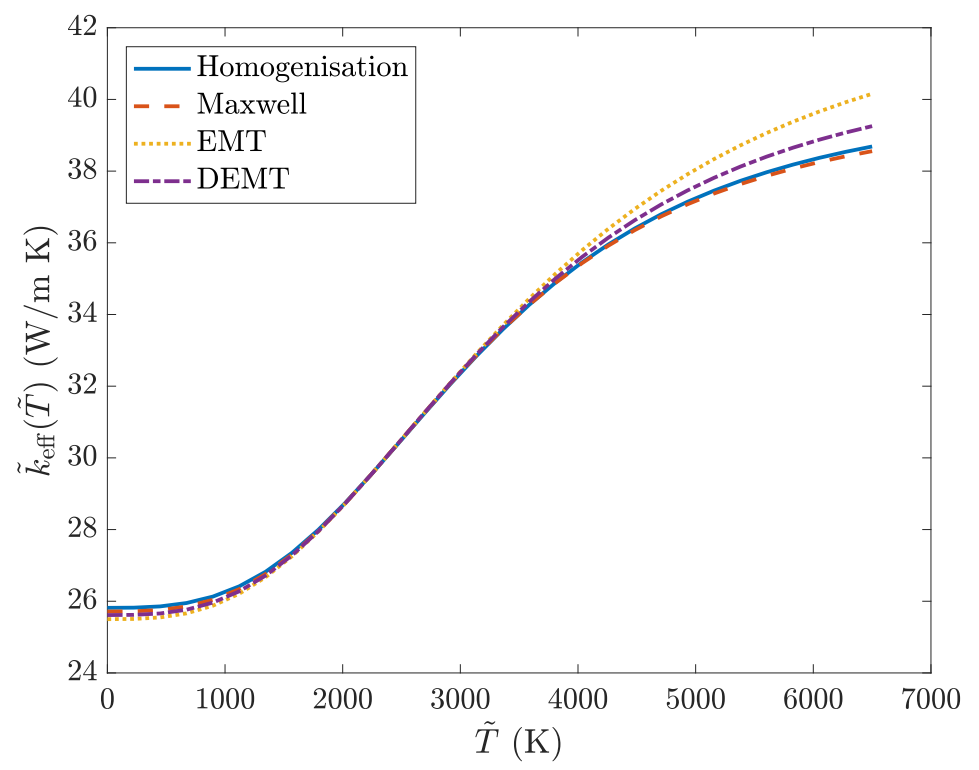

(a) Void radius $0.01 \mathrm{~m}$.

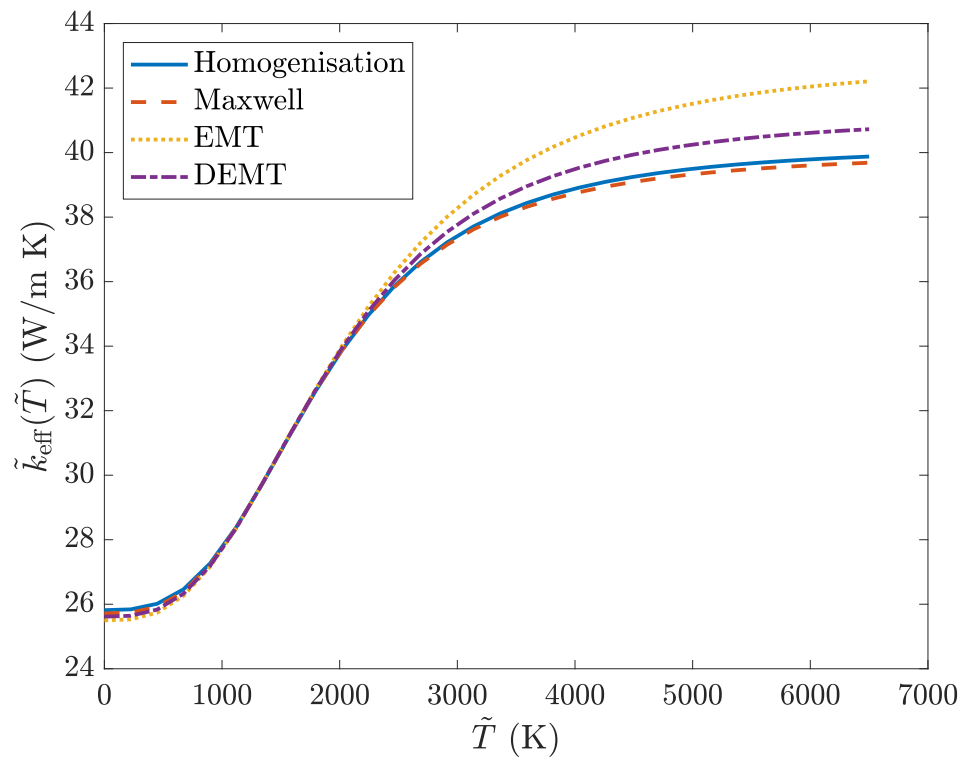

(b) Void radius $0.05 \mathrm{~m}$.

Figure 5. Comparison between homogenisation (this work), Maxwell, EMT and DEMT (Kiradjiev et al. [10]). 


\section{Discussion and conclusion}

We have explored the influence of radiative effects on the thermal transfer within a solid matrix with gaseous perforations or voids. The presence of a nonlinear, integral boundary condition in the governing equations posed a computational complication, however with careful consideration of the multiple scales methodology, we used Chapman and McBurnie's 4, 12 extension of the standard homogenisation technique to treat the nonlocal condition. From this, we derived a homogenised model and effective thermal conductivity tensor. We compared the homogenised solution with that obtained directly and observed that the approximation error of the former decreases with microstructure period $\delta$, thereby verifying that our homogenisation model can accurately approximate conductive-radiative transfer within the domain. By noting the computational costs for each of the solutions, we conclude that as the number of voids increases, obtaining a solution directly becomes intractable and, in fact, the homogenised solution is a very good approximation to such a solution.

Interestingly, the conduction-radiation effective conductivity tensor consists of a conductive term plus a radiative term. The separation of the two thermal contributions in (5.30) allows us to qualitatively understand their respective effects. It is clear from the presence of the third power of homogenised temperature $T_{0}$ in the radiative term of 5.30

that as $T_{0}$ increases, radiation plays a larger role in determining the effective conductivity; however, we deduced numerically that the conductivity saturates for large temperatures, therefore the radiation does not dominate over conduction, rather the conducting solid is the limiting factor.

\section{Acknowledgements}

This work is supported by the EPSRC Centre for Doctoral Training in Industrially Focused Mathematical Modelling (EP/L015803/1). It is also supported by the Electrical Conditions and their Process Interactions in High Temperature Metallurgical Reactors (ElMet/247791) project which is a cooperation between NORCE, The Norwegian University of Science and Technology, and the companies Elkem and Eramet, and financially supported by The Research Council of Norway and the two companies. C Rooney thanks Svenn Anton Halvorsen and Manuel Sparta for their insight throughout this project. We also thank Ellen Luckins for the proof given in Appendix A, and Mo Dalwadi, Jon Chapman and Kristian Kiradjiev for helpful discussions. Finally, we thank the reviewers for their constructive comments.

\section{Appendix A The radiation-dominated regime}

Consider (3.4) and write $u(\mathbf{y})=T^{4}(\mathbf{y})$; thus, for $\mathbf{y} \in \Gamma$ we write

$$
u(\mathbf{y})=\int_{\Gamma} u\left(\mathbf{y}^{\prime}\right) F\left(\mathbf{y}, \mathbf{y}^{\prime}\right) \mathrm{d} S\left(\mathbf{y}^{\prime}\right) .
$$

Assume that $u$ is continuous and $\Gamma$ is convex. We claim that the only solution is $u=$ constant. 
Proof Assume that $u$ is non-constant. As $u$ is continuous, it is bounded by a maximum value $u_{\max }$ and equals $u_{\max }$ on at least one point on $\Gamma$. As $u$ is non-constant, then thejre exists a subset $G \in \Gamma$ such that for $\mathbf{y} \in G$,

$$
u(\mathbf{y})<u_{\max } .
$$

However, since $u$ is a solution to A 1, it satisfies

$$
\begin{aligned}
u(\mathbf{y})=u_{\max } & =\int_{\Gamma} u\left(\mathbf{y}^{\prime}\right) F\left(\mathbf{y}, \mathbf{y}^{\prime}\right) \mathrm{d} S\left(\mathbf{y}^{\prime}\right), \\
& =\int_{\Gamma \backslash G} u_{\max } F\left(\mathbf{y}, \mathbf{y}^{\prime}\right) \mathrm{d} S\left(\mathbf{y}^{\prime}\right)+\int_{G} u\left(\mathbf{y}^{\prime}\right) F\left(\mathbf{y}, \mathbf{y}^{\prime}\right) \mathrm{d} S\left(\mathbf{y}^{\prime}\right), \\
& <\int_{\Gamma \backslash G} u_{\max } F\left(\mathbf{y}, \mathbf{y}^{\prime}\right) \mathrm{d} S\left(\mathbf{y}^{\prime}\right)+\int_{G} u_{\max } F\left(\mathbf{y}, \mathbf{y}^{\prime}\right) \mathrm{d} S\left(\mathbf{y}^{\prime}\right), \\
& =u_{\max } \int_{\Gamma} F\left(\mathbf{y}, \mathbf{y}^{\prime}\right) \mathrm{d} S\left(\mathbf{y}^{\prime}\right), \\
& =u_{\max }
\end{aligned}
$$

using (2.3). This is a contradiction, therefore $u$ must be constant.

\section{References}

[1] Allaire, G., and El Ganaoui, K. Homogenization of a conductive and radiative heat transfer problem. Multiscale Modeling \& Simulation 7, 3 (2009), 1148-1170.

[2] Allaire, G., ANd Habibi, Z. Homogenization of a conductive, convective, and radiative heat transfer problem in a heterogeneous domain. SIAM Journal on Mathematical Analysis 45, 3 (2013), 1136-1178.

[3] Allaire, G., And Habibi, Z. Second order corrector in the homogenization of a conductiveradiative heat transfer problem. Discrete and Continuous Dynamical Systems-Series B 18, 1 (2013), 1-36.

[4] Chapman, S. J., And McBurnie, S. E. Integral constraints in multiple-scales problems. European Journal of Applied Mathematics 26, 5 (2015), 595-614.

[5] Chapman, S. J., Shipley, R. J., And Jawad, R. Multiscale modeling of fluid transport in tumors. Bulletin of Mathematical Biology 70, 8 (2008), 2334.

[6] Dalwadi, M. P., Griffiths, I. M., And Bruna, M. Understanding how porosity gradients can make a better filter using homogenization theory. Proceedings of the Royal Society A: Mathematical, Physical and Engineering Sciences 471, 2182 (2015), 20150464.

[7] Francl, J., And Kingery, W. D. Thermal conductivity: Ix, experimental investigation of effect of porosity on thermal conductivity. Journal of the American Ceramic Society 37, 2 (1954), 99-107.

[8] Howell, J. R., Menguc, M. P., And Siegel, R. Thermal radiation heat transfer. CRC press, 2015.

[9] Jones, E., Oliphant, T., Peterson, P., et Al. SciPy: Open source scientific tools for Python, 2001.

[10] Kiradjiev, K. B., Halvorsen, S. A., Van Gorder, R. A., and Howison, S. D. Maxwell-type models for the effective thermal conductivity of a porous material with radiative transfer in the voids. International Journal of Thermal Sciences 145 (2019), 106009.

[11] Luikov, A. V., Shashkov, A. G., Vasiliev, L. L., and Fraiman, Y. E. Thermal conductivity of porous systems. International Journal of Heat and Mass Transfer 11, 2 (1968), 117-140. 
[12] McBurnie, S. E. Sound propagation through bubbly liquids. PhD thesis, Oxford University, 2010.

[13] Modest, M. F. Radiative heat transfer. Academic Press, 2013.

[14] Ockendon, J. R., Howison, S., Lacey, A., And Movchan, A. Applied partial differential equations. Oxford University Press, 2003.

[15] Palencia, E. S. Non-homogeneous media and vibration theory. Springer-Verlag, 1980.

[16] Pasternak, E., and Mühlhaus, H. B. Generalised homogenisation procedures for granular materials. In Mathematics and Mechanics of Granular Materials. Springer, 2005, pp. 199-229.

[17] Pavliotis, G., And Stuart, A. Multiscale methods: averaging and homogenization. Springer Science and Business Media, 2008.

[18] Rathgeber, F., Ham, D. A., Mitchell, L., Lange, M., Luporini, F., McRae, A. T. T., Bercea, G.-T., Markall, G. R., and Kelly, P. H. J. Firedrake: automating the finite element method by composing abstractions. ACM Transactions on Mathematical Software (TOMS) 43, 3 (2017), 24.

[19] Rooney, C. M. Homogenisation of the electrothermal behaviour of granular material. $\mathrm{PhD}$ thesis, University of Oxford, 2019.

[20] Rosseland, S. Theoretical astrophysics; atomic theory and the analysis of stellar atmospheres and envelopes. Clarendon Press, Oxford, 1936.

[21] Schatz, J. F., And Simmons, G. Thermal conductivity of Earth materials at high temperatures. Journal of Geophysical Research 77, 35 (1972), 6966-6983.

[22] Spinnler, M., Winter, E. R., And Viskanta, R. Studies on high-temperature multilayer thermal insulations. International Journal of Heat and Mass Transfer 47, 6-7 (2004), $1305-1312$.

[23] Tilnonen, T. Stefan-Boltzmann radiation on non-convex surfaces. Mathematical Methods in the Applied Sciences 20, 1 (1997), 47-57.

[24] Zhao, C. Y., Lu, T. J., Hodson, H. P., And Jackson, J. D. The temperature dependence of effective thermal conductivity of open-celled steel alloy foams. Materials Science and Engineering: A 367, 1-2 (2004), 123-131.

[25] Zhou, Q., Zhang, H. W., And Zheng, Y. G. A homogenization technique for heat transfer in periodic granular materials. Advanced Powder Technology 23, 1 (2012), 104-114. 